\title{
Intrathecal infusions for intractable cancer pain: A qualitative study of the impact on a case series of patients and caregivers
}

\author{
Philippa Hawley FRCPC ${ }^{1}$, Elizabeth Beddard-Huber RN MSN ${ }^{1}$, Cameron Grose BSc ${ }^{2}$, \\ William McDonald FRCPC ${ }^{3}$, Daphne Lobb MD ${ }^{3}$, Louise Malysh RN MSN ${ }^{3}$
}

\begin{abstract}
P Hawley, E Beddard-Huber, C Grose, W McDonald, D Lobb, L Malysh. Intrathecal infusions for intractable cancer pain: A qualitative study of the impact on a case series of patients and caregivers. Pain Res Manage 2009;14(5):371-379.
\end{abstract}

BACKGROUND: The need for intrathecal infusion in a palliative care setting is infrequent. Despite established efficacy, safety and cost effectiveness, this is considered an 'extraordinary measure' in Canada. Patients requiring this approach are not typical palliative care patients, having shorter and more uncertain life expectancies.

OBJECTIVES: The present study is a qualitative exploration of the impact of intrathecal pump implantation on cancer patients, and also the impact of the intervention on the staff caring for those patients.

METHODS: Palliative care unit patients who received an implanted intrathecal pump or dome catheter for intractable cancer pain participated in multiple semistructured interviews. Doctors and nurses caring for each patient were also interviewed. Interviews were recorded and analyzed for themes. The study terminated when saturation was reached.

RESULTS: Six patients participated, with up to three interviews each. Twenty-four staff interviews took place. Patients' hopes and expectations were not always fully met, but the infusions had a profound positive effect on quality of life. Patients expressed anxiety about dependence on the device, and also on a few highly skilled individuals. Staff interviews revealed a significant impact on the 'culture' of the palliative care unit. Clear communication of the rationale for infusion was very important, as was regular education about infusion management.

CONCLUSIONS: Implanted intrathecal infusion devices are a necessary part of a tertiary level cancer pain management service for the unfortunate minority with intractable pain. Practical recommendations for care are made for palliative care programs contemplating offering intrathecal infusions.

Key Words: Anesthesia; Canada; Cancer; Intrathecal; Pain; Palliative care

\section{Les infusions intrathécales pour traiter les douleurs cancéreuses réfractaires : Une étude qualitative des répercussions sur une série de patients et de soignants}

HISTORIQUE : Les infusions intrathécales sont rarement utilisées en soins palliatifs. Malgré une efficacité, une innocuité et une rentabilité établies, elles sont considérées comme une " mesure extraordinaire " au Canada. Les patients qui ont besoin de ce traitement ne sont pas des patients en soins palliatifs classiques, car leur espérance de vie est plus courte et plus incertaine.

OBJECTIFS : La présente étude est une exploration qualitative des répercussions de l'implantation d'une pompe intrathécale chez des patients cancéreux, de même que des répercussions de l'intervention sur le personnel qui s'occupe de ces patients.

MÉTHODOLOGIE : Les patients de l'unité de soins palliatifs à qui on avait implanté une pompe intrathécale ou un cathéter courbe pour traiter des douleurs cancéreuses réfractaires ont participé à de multiples entrevues semi-structurées. Les médecins et les infirmières soignant chaque patient ont également participé aux entrevues. On a enregistré et analysé les entrevues pour en dégager les thèmes. L'étude a pris fin une fois le point de saturation atteint.

RÉSULTATS : Six patients ont participé, et chacun a réalisé jusqu'à trois entrevues, tandis que le personnel a participé à 24 entrevues. Les espoirs et les attentes des patients n'étaient pas toujours entièrement respectés, mais les infusions avaient un effet positif profond sur la qualité de vie. Les patients ont exprimé leur anxiété à l'égard de la dépendance au dispositif et de quelques personnes très compétentes. Les entrevues auprès du personnel ont révélé une répercussion importante sur la « culture » de l'unité de soins palliatifs. Il était très important de communiquer clairement la raison d'être de l'infusion et de fournir une formation régulière sur sa prise en charge.

CONCLUSIONS : Les dispositifs d'infusion intrathécale sont un élément nécessaire des services de prise en charge des douleurs cancéreuses au sein de la minorité malchanceuse souffrant de douleurs réfractaires. On présente des recommandations pratiques à l'intention des programmes de soins palliatifs qui envisagent d'offrir des infusions intrathécales.

space. These procedures are collectively considered to be step 4 of the World Health Organization pain ladder (2). In practice, neuraxial infusions are the preferred modality because they are reversible, usually effective and significantly cheaper than stimulators.

The choice of route of neuraxial administration of medications has been clarified since the first intrathecal infusions were described by Wang et al in 1979 (3). Extensive clinical experience and a small number of comparative trials over the past 40 years have led to preferred choices of route under certain circumstances (4-14). Epidural catheters are straightforward to ered into either the epidural or intrathecal (subarachnoid)

${ }^{1}$ Pain and Symptom Management/Palliative Care Program, BC Cancer Agency, Vancouver; ${ }^{2}$ University of British Columbia, Northern Medical

Program, Prince George; ${ }^{3}$ St Paul's Hospital Palliative Care Unit, Vancouver, British Columbia

Correspondence: Dr Philippa Hawley, BC Cancer Agency, 600 West 10th Avenue, Vancouver, British Columbia V5Z 4E6.

Telephone 604-877-6000 ext 2707, fax 604-877-0585, e-mail phawley@bccancer.bc.ca 
insert in even very sick patients, but are prone to migration and require approximately 10 times the dose of drug than the intrathecal route. They are unsuitable for attachment to implantable reservoirs because of the volumes of drug required. Epidurals are generally preferred over the intrathecal route when analgesia is required for a short period - for example, while awaiting a surgical procedure, or when a patient is bedbound and very near the end of life. Intrathecal catheters are less prone to migration and are more suitable for long-term use. Catheter tip granulation can occur with both routes, but is less common with intrathecal catheters. Infection and bleeding are potential complications of both routes; however, infection of an intrathecal catheter has more potential for serious harm than infection of an epidural infusion. A continuous, percutaneously accessed system also restricts bathing and other activities; thus, fully implanted systems are preferred for ambulatory patients or if an infusion is expected to be required for more than a few weeks.

Percutaneously accessible subcutaneous 'dome' catheters or implantable pumps have been available for continuous intrathecal infusion for some time, and a large amount of clinical experience with these devices has now been gained. Their effectiveness has been clearly documented in large case series $(15,16)$ and one randomized trial (17). By 1999, they were (and still are) considered standard therapy for cancer patients whose pain had not responded to step 3 of the World Health Organization pain ladder $(18,19)$. However, in Canada and many other countries, this intervention is rarely available. There are many reasons for this variation in clinical practice, including financial concerns, unfamiliarity with the intervention and lack of anesthesia support in palliative care settings. Most Canadian palliative care units (PCUs) care for 100 to 300 admissions per year. The need for intrathecal or epidural infusions is relatively rare (estimated at less than $5 \%$ as mentioned previously) and, in those who need this intervention, it is also relatively rare that prognosis is sufficiently long to justify an implantable device (less than 1\%) either financially or in terms of the patient's ability to tolerate the procedures required for insertion. Intrathecal infusions are therefore considered 'extraordinary measures', conflicting with traditional minimally invasive palliative care and hospice philosophy. In British Columbia, implantable intrathecal infusion pumps are only able to be implanted in three centres, and the expensive equipment is not covered by the provincial Medical Services Plan. In some provinces of Canada, they are completely unavailable.

Despite the infrequent need, these rare patients with intractable pain experience extreme suffering. Patients are often transferred from distant locations to access the specialized team; therefore, staff at the referral site rarely get an opportunity to get to know the patients well before the intrathecal trial. They have very complex pain syndromes and are sometimes first referred to palliative care specialists in the setting of taking high doses of opioids and other adjuvant medications, often with sedation and delirium, which preclude a good psychological assessment (20).

Since late 2002, a joint initiative between the BC Cancer Agency and St Paul's Hospital (Vancouver, British Columbia) has allowed patients from the lower mainland of British Columbia suffering from intractable cancer pain to be offered a trial of intrathecal therapy on the PCU at St Paul's Hospital. The trial is followed (if successful) by implantation of a device to maintain the infusion over an extended period, expected to be the rest of the patient's life. Consistent with international consensus guidelines, if life expectancy is over three months a fully implanted pump and reservoir system is used. If life expectancy is less than three months, but more than a few weeks, a dome system is used, with an external pump. The anesthesia team had extensive experience with intrathecal pump placement and management in patients with noncancer pain before the first palliative care patient was treated, and had implanted seven cancer patients in the three years before the present study. Experience with the pumps varied from spectacular long-term success to minimal overall benefit, the latter usually being due to other severe symptoms of cancer emerging after pump implantation.

Those patients who did receive an implanted pump needed to be followed closely by palliative care and anesthesia staff, and the presence of the pumps added a level of complexity to their care needs due to the requirement for regular refills (approximately monthly) and lack of familiarity with the devices at other institutions.

Some concerns were raised by PCU staff during this time about how best to care for these rare but highly complex patients with extreme pain and suffering. We also recognized that the intrathecal program had had a major impact on the running of the unit, with some conflict between staff around appropriateness of the intervention in the palliative care setting.

The present qualitative study was designed to first determine the impact of intrathecal infusions on patients with intractable cancer pain and, second, to explore how caring for such patients impacts the staff in a palliative care environment.

\section{METHODS}

The present study was a qualitative exploration of the experience of consecutive intrathecal patients and their care providers. Study patients were those considered for long- or medium-term intrathecal analgesia for severe cancer-related pain with a fully implantable system. Patients near the end of life who received intrathecal or epidural infusions through a temporary percutaneous catheter were not included, although there were very few of these during the study period. All patients were admitted to the PCU at St Paul's Hospital for assessment before the intrathecal trial. Doses and choice of drugs infused varied widely among patients and were optimized individually, consistent with current best international practice (21). Equipment was supplied by Medtronic Inc, USA, and paid for by the BC Cancer Agency.

Patients were required to have tried multiple opioids by oral and parenteral routes, and adjuvant analgesics as appropriate for the pain diagnosis or diagnoses. Patients had access to methadone, lidocaine and ketamine infusions. All patients had access to patient and family counselling support through the BC Cancer Agency before admission, although not all took advantage of it.

Physicians and nurses were included in the study if they provided direct care to a study patient.

Semistructured interviews (Tables 1 and 2) were conducted by a research nurse or research assistant who was not 
TABLE 1

Interview guideline questions for patients

Pain experience

Could you describe what your pain was like before receiving an intrathecal infusion?

\section{Quality of life}

What drawbacks or concerns do you see in having an intrathecal infusion?

What benefits do you see?

What additional support do you think you will need?

Hope/expectation

What are your expectations around the intrathecal infusion?

\section{Effectiveness}

Could you describe if there has been any difference in the nature of your pain since you began having the intrathecal infusion?

Comfort level with device

Do you have any concerns about the safe use of an intrathecal infusion in administering pain medications?

involved with patient care. Interviews were tape-recorded and transcribed verbatim. Up to three interviews were conducted with each patient - an initial interview between the trial of the intrathecal infusion and the implant, a second interview four to six weeks after implantation, and the last at four to six months after implantation. Questions directed at patients focused on the impact that the intrathecal infusion had on their pain experience. Written questionnaires concerning pain and symptom management, and perceived quality of life, were also completed daily by the patients for seven days after implantation and then at monthly intervals until death.

Two nurses and two physicians directly involved in care of each study patient were interviewed separately. Questions directed at health care providers focused on what it was like to care for a patient.

The six or seven transcripts generated by each case were analyzed and interpreted as a unique stand-alone case and then cross-referenced with those of other patients as the study proceeded, to identify recurring themes and levels of saturation.

The present study was approved by the Ethics Review Boards of St Paul's Hospital (Providence Health) and the BC Cancer Agency in conjunction with the University of British Columbia's Behavioural Research Ethics Board.

\section{RESULTS}

\section{Description of patients}

A total of seven patients participated from October 2005 to saturation of themes in October 2007. Six patients had successful intrathecal trials, leading to four fully implantable pumps and two dome systems implanted. One patient withdrew from the study because of rapid medical deterioration that led to the decision not to proceed with the pump implant. All patients received intrathecal bupivocaine in combination with either fentanyl or hydromorphone. The doses and combinations were titrated individually according to analgesic response and side effects. No study patients received clonidine or other agents.

Establishment of effectiveness of intrathecal infusion was not the purpose of the present study, and the number of patients was small; therefore, the pain outcomes are described here but were not analyzed statistically. All patients rated their

\section{TABLE 2}

Interview guideline questions for health care providers Decision-making process

How typical is this patient to other patients you have had with intrathecals? Please tell me about the introduction of the intrathecal for this patient. Changing culture in a palliative setting

In retrospect, was there anything more that could have been done to accommodate this intrathecal patient on the palliative care unit?

\section{Patient assessment}

Have you noticed any changes in the patient's behaviour or mobility since starting on the intrathecal infusion?

\section{Quality of life}

Please comment on the initial effectiveness of the intrathecally infused medication to manage this patient's pain.

Have you noticed any changes in the patient's management of pain and related symptoms since being on the intrathecal infusion?

\section{Education}

Was there any intrathecal-specific training provided for the health care workers who provide clinical care to this intrathecal patient?

\section{Expectation}

How long do you anticipate this patient will receive their medication administered by an intrathecal infusion?

\section{Comfort level with device}

Do you have any concerns regarding the accurate and safe administration of pain medication by an intrathecal infusion for this patient?

pain as being at least 9 on a 0 to 10 numerical scale or 'very severe' before implantation. After commencement of intrathecal infusion, three of the patients rated their average pain at 1 , one patient at 2 , one at 3 and one patient who was not able to use the numerical scale rated her pain as 'moderate'. Patients had difficulty rating their 'quality of life' both before and after the implants. For those who were able to complete the Edmonton Symptom Assessment Scale before intrathecal insertion, scores ranged from 34.5 to 40 and the postinsertion scores ranged from 22 to 33 (maximum of 90). The Palliative Outcome Scale $(22,23)$ scores before intrathecal insertion ranged from 12 to 19 and the scores afterward ranged from 10 to 11 (maximum of 40 ).

All six of the patients in the study were discharged from the PCU. Four were discharged home and two were transferred to a residential hospice. Survival after implantation was between 11 weeks and 12 months. Three patients returned to the unit due to cancer progression and one (who had a dome-accessed intrathecal catheter) due to staphylococcal meningitis.

Life expectancy was difficult to predict. One of the two patients who received a dome implant (life expectancy at the time of implant estimated at less than three months) actually survived for eight months, having had the implant removed after five months because of bacterial meningitis. She had a very prolonged PCU admission and, in hindsight, a fully implantable system would have been a better choice for her, and would probably have avoided the meningitis. For the four patients who received fully implantable devices, survival was 11 weeks, four months, 10 months and 12 months.

Themes identified from patient interviews (Table 3)

Pain experience - coping with disease: The pain experience for these patients before the intrathecal implant was devastating. It impacted all aspects of their lives and was overpowering in its magnitude. 
TABLE 3

Case series

\begin{tabular}{|c|c|c|c|c|c|c|}
\hline Case & Age, years & Sex & Diagnosis & Implant pump & Implant dome & Death \\
\hline A & 22 & Female & Stage III invasive squamous cell cancer of the vagina & & October 2005 & June $2006^{*}$ \\
\hline C & 47 & Male & Metastatic thyroid cancer & May 2006 & & March 2007 \\
\hline$E$ & 45 & Male & Lumbar osteoblastoma & January 2007 & & January 2008 \\
\hline $\mathrm{F}$ & 67 & Female & Recurrent metastatic uterine cancer & & July 2007 & September 2007 \\
\hline
\end{tabular}

*Dome removed in February 2006 due to staphylococcal meningitis

What's life living in pain? There's no meaning; like you just drag yourself and eating all these pills, everywhere you go you feel pain. There's no life. (Patient A, Interview 1)

It didn't seem that there was anything I could do to get relief...right...I couldn't sit I couldn't stand, I couldn't do anything that brought me any relief whatsoever. (Patient B, Interview 1)

I just don't want to put up with it anymore. The pain was that horrible. (Patient B, Interview 1)

Like I never even really thought of anything except for that particular pain. (Patient D, Interview 2)

Hope and expectations: The intrathecal infusion was seen as a 'last resort' or 'last chance' that sparked many hopes and expectations. Expectation is defined as something to look forward to and hope as the combination of expectation and desire. For these patients, the intrathecal infusion represented hope for a life without pain.

I expect to see myself able to walk and able to sit down in a chair...just like a normal person. (Patient A, Interview 2)

My ambition would be for complete absence of a life being controlled by pain. (Patient B, Interview 2)

Hopefully that what it is going to enable me to do is to carry on more of a regular life. (Patient D, Interview 2) goals.

Independence and a sense of control were also patient

To make any adjustments on my own without bugging the doctor all the time...I am hoping this will alleviate that feeling that I'm being a pest because I needed some help. (Patient B, Interview 1)

Just to test out to see if I am going to be a bit helpless to be away from, you know, the secure area of my house and all the nurses that come and see me...to see if I can just kind of do it on my own for a week. (Patient D, Interview 2)

Effectiveness: All patients reported that the intrathecal implant was helpful, sometimes dramatically so.
Well it gets me to live more of a regular life. You know for what life I have left. (Patient D, Interview 2)

At least be able to get up without pain which I wasn't able to do before, it was so hard to walk just around my house and not be in pain. (Patient F, Interview 1)

Without intrathecal, quite frankly my pain would be so severe; I would be around 9 all the time... and now 5 is the average and it goes down to 2 , and in this position sitting I feel like it is $0 \ldots$ yeah...it's unbelievable. So this having the pump has saved me a lot of trouble, from trouble from terrible nights. (Patient C, Interview 2)

Right now I am sitting down on a seat that uh I wasn't able to do six months ago. (Patient D, Interview 2)

I was able to get up off the bed and walk. (Patient A, Interview 2)

However, the hope and expectations of some of the patients were not fully met.

How come it doesn't take my pain away? All of it...like, how come I am still in pain? (Patient A, Interview 2)

The impression was that it would totally get rid of the pain...um...which it hasn't done, but then I don't know whether that was expecting too much or whether the doctors expected it to get rid of the pain entirely. (Patient F, Interview 1)

Comfort level with the device: Patients were comfortable with having the implant, although some were anxious about possible device failure.

I think I feel quite comfortable with it. (Patient B, Interview 2)

I mean if it stopped then I'd be in big $\mathrm{s}^{* * *}$. (Patient D, Interview 2)

If I have an emergency outside my home jurisdiction which is Vancouver...how...sometimes I wonder if I would get easy access to somebody who understands the intrathecal pump. (Patient C, Interview 2) 


\section{Themes identified from staff interviews (Table 4)}

Staff varied in their years of experience, with some working for over 30 years, while others were new to the profession. Twelve nurses and 13 physicians were interviewed. Table 4 documents the major themes and subthemes that emerged in the analysis of the interviews. The themes expressed by staff included anxiety associated with the delivery of high-intensity medical care in a palliative setting, temporary change in code status, patient acuity and workload - all of which depart from the traditional palliative care environment. They expressed concerns regarding sustainability of limited resources, health care providers' ethical and moral responsibility, and staff education.

Caring for patients with intrathecal infusions on a PCU altered the culture of the unit and was met with varying opinions based on personal values and beliefs. Some nurses believed that the procedure was too invasive for a 'palliative patient', while others believed it was their moral responsibility to offer care for these individuals.

Decision-making process: Concern about the decisionmaking process around the intrathecal implant was a common concern among nurses, especially those who had not cared for the patients on the ward before the intrathecal trial. Usually, the decision to admit a patient for an intrathecal trial was made by doctors, and patients were not known to the anesthesia or palliative care staff before admission. It was recognized that the patients had unusually complex or treatment-resistant pain. It was also recognized that, for most of the patients, it was not possible to carry out a long or detailed psychological assessment before the intrathecal trial, when they presented in a pain crisis with cognitive or concentration impairment as a result of severe pain and/or medication side effects.

I would hope that they [patients] would get again a thorough assessment both from nursing, from doctors and from a psychologist or counsellor, or social worker and family as well and that time be spent teaching with the patient, with the family perhaps by one of the intrathecal nurses. (Nurse 2 for Patient C)

...That everything is addressed about a person, their pain and where they come from and maybe a little more communication with the staff, the nursing staff and the physicians and the pain service team about the people that we send for these. Because often I feel we do it too late, way too late. (Nurse 1 for Patient E)

...He had tried every available drug to help with his pain and nothing was working, and he knew that there was nothing else for him to try. (Nurse 1 for Patient C)

Yeah I think for some people who have ended up with an intrathecal, I think that they were not good candidates for it. (Nurse 1 for Patient B)

Does this person really understand what they agreed to do? I mean that is huge. (Nurse 2 for Patient C)

The palliative physicians recognized their moral and ethical responsibility to these patients, and responded to the interviewer's questions by saying:

\section{TABLE 4}

Emergent categories and themes

Patients' perspective
1. Pain experience - coping with disease
2. Quality of life
i. Grief and loss
ii. Support
3. Hope/expectation
i. Patient reflection
ii. Disappointment
4. Effectiveness
5. Comfort level with device
Health care providers' perspective
1. Decision-making process
i. Criteria/patient selection
ii. Desperate situation
iii. Burden versus benefit
iv. Ethical and moral responsibility
2. Communication
3. Changing culture in a palliative setting - staff anxiety due to conflicting
values
4. Change in code status
5. Complex patients - patient assessment
6. Quality of life for patients
7. Workload
8. Education
9. Accessibility/sustainability
10. Hope and expectation
11. Comfort level with device - trust

I think if we could have everything black and white and a guarantee that this is going and it will all be worth it. Sure it makes life, work, easier but most of palliative care is grey so you have to have a level of comfort working in the grey. It can be anxiety-provoking so I think it is both from a discomfort and anxiety, and a sort of questioning about whether it is helpful. (Doctor 2 for Patient A)

It is really a medical decision based on the need for good pain management. (Doctor 1 for Patient B)

Quality of life for patients: Staff appreciated the immense positive impact on patients' quality of life.

He is much happier, more gregarious, he is very animated anyway but with the intrathecal he just keeps saying 'Oh you gave me back my life again'. He has just been so elated about this experience. (Nurse 1 for Patient C)

He was able to get into the chair, he was giddy, just amazing, getting out to the balcony was huge....getting to go to the bath was huge, you know it was fabulous. (Nurse 2 for Patient D)

She was up and mobile, providing all her own personal care, completely independent and that was the goal. (Nurse 1 for Patient F)

Communication: Nurses identified the need for good communication among the care team members. 
Get to know the patient, get to know their pain a little bit more so that it makes the after-assessment a little more pertinent. (Nurse 2 for Patient C)

We have to establish our communication lines... who do I call if this patient is on this unit, who do I call if there is a problem. (Nurse 2 for Patient D)

I think the nursing staff really need to have that information very clear for them so they can have a little more security and realize that this, this is a good choice for this patient. (Nurse 1 for Patient D)

Changing culture: The change in code status from Do Not Resuscitate (DNR) to resuscitate in the event of cardiac and/or respiratory arrest ('full code') created anxiety among the staff, especially for those who had worked exclusively on the PCU for a long time.

The fact that the person is a full code for the $48 \mathrm{~h}$ postinsertion and that goes against the whole philosophy which is you need to be in agreement that you are a DNR before you come to the unit. It puts a lot of strain I think on many nurses, and I am sure on doctors too. (Nurse 2 for Patient A)

I have worked on floors before that all of my patients are full codes so for me I do not have the fear. (Nurse 2 for Patient A)

When we have someone on the ward that is a full code everyone sort of feels it. (Nurse 1 for Patient A)

The commencement of the intrathecal program coincided with the opening of a number of local residential hospices, with less complex patients being cared for in a hospice rather than on the PCU. The resulting increase in acuity of patients on the PCU was therefore not just due to the intrathecal program. The increase in acuity did, however, cause some anxiety in staff, and the intrathecal program was a natural focus for some of this anxiety. This was more difficult for some of the more experienced nurses, who were more attuned to the noninterventional 'culture' of hospice, and less comfortable with the change than some of the more recent graduates.

We are more acute palliative medicine than hospice care now. It has been a real shift and it has been something that some people on the ward have struggled with in terms of understanding just how it fits into the work that they have chosen to do. (Nurse 1 for Patient D)

The intensity of the care took away from the peaceful, more quiet atmosphere we were trying to maintain on the unit...the person is full code which is not something that we want to see happening on a palliative care unit for many reasons. (Nurse 2 for Patient C)

I just graduated last year...my feeling is that if it works and they still have a good amount of life to live and that we can manage it...if we can deliver it safely and monitor it well then I think it is great, I think that it is wonderful that we could do that for people even if it is a bit more invasive than we are used to. (Nurse 1 for Patient B)

Complex patients - patient assessment: It was noted that some patients often have very complex pain syndromes. Their physical pain may be well managed with the intrathecal infusion, but the emotional, psychosocial, spiritual and intellectual aspects of their pain still needed to be addressed.

So he is very complex from the psychosocial point of view as well as from his pain. (Nurse 2 for Patient C)

These patients are the most complex...in the top $2 \%$ of complexity of their pain management so they often have a huge psychosocial and family component to their illness and their pain...there is depression, anxiety, the fact that they have been dealing with doctors and nurses for a long time. (Nurse 1 for Patient D)

The nurses were getting to know him a little bit and were getting quite good at sorting out when he had real pain and when he was looking for attention or relief from anxiety. (Doctor 1 for Patient E)

So I think there were three different types of pain that he was experiencing. (Doctor 2 for Patient E)

Assessing her pain has always been difficult, and you hate to judge and you hate to say it is just her being hypersensitive...she has pain, right? (Nurse 2 for Patient F)

In other patients, the pain was less complex, but responded poorly to other treatments.

She was pretty clear on what did work and what did not work. (Nurse 1 for Patient B)

Everything was just out there, how he was feeling, what was going on was very prominent...it was just evident how improvements when they came it was just easily evident to see him and speak to him about that. And he did have just a good response. (Doctor 2 for Patient C)

Workload: The change in code status and the acuity of these patients demanded increased monitoring and impacted the workload on the unit.

It takes up a lot more time...often times these people have family members that are there and are quite distraught witnessing this so we spend a lot of time doing education, teaching around what they should expect. (Nurse 2 for Patient A)

There is certainly a lot more involved. There is a lot more assessment. There is a lot more that can go wrong with them. Sort of a higher anxiety with them certainly for the first little while anyway. (Nurse 1 for Patient A) 
Instead of three nurses per shift you need four...there is a finite pool of people that are qualified to work on the unit. (Nurse 1 for Patient D)

Education: Health care providers recognized the need for staff and patient education as a means to reduce staff anxiety when caring for the present patient population. Nurses expressed anxiety related to lack of training and education on the intrathecal infusion. Throughout the study period, educational initiatives were performed and some of the comments reflected these improvements. However, there is still a need for ongoing education to ensure that new and casual staff members are comfortable caring for a patient with an intrathecal infusion.

When we first started doing intrathecals they just started appearing on the ward without any consultation with nursing, and any education for nurses at that time and without any planning in terms of having extra nurses on because when somebody just has a fresh intrathecal they need very, very close, almost one-to-one monitoring and that was not even considered at first. It took a long time; I mean it is happening now more. (Nurse 2 for Patient C)

If I can look around on this shift right now...I do not know that there is enough education support there and I feel that inexperienced staff, comfort level aside, I do not even know if they are aware of how uncomfortable they should feel. (Nurse 2 for Patient D)

I thought that it was kind of scary for me...but it was recommended to read those brochures and pamphlets, and we also had the intrathecal pump itself...I wish I had more inservice. (Nurse 2 for Patient B)

We have got a real pathway now. (Nurse 1 for Patient A)

We literally try as a goal to only have one patient at a time and that is for all of our sakes. (Doctor 2 for Patient D)

Accessibility and sustainability: As health care providers developed trust of the device and of the specialists involved, the level of anxiety among nursing staff declined. However, the concern regarding sustainability of the limited resources was raised. The need for transfer to a different hospital delayed the process in some cases.

Nursing a patient is not just having a bed, it is having the professional staff that mattered to look after that patient. (Nurse 2 for Patient C)

Being a young woman who wanted to take every chance she had at life she chose to take chemotherapy, which meant that she was not able to get the intrathecal because the chemotherapy has to be done here [BC Cancer Agency] and the intrathecal cannot be done here. (Doctor 1 for Patient A)
Hope and expectations: The hopes and expectations expressed by the health care providers focused on enhancing the patients' quality of life and reflected the patients' comments.

I was just incredibly hopeful that she would receive the pain relief that she deserved to have. (Nurse 2 for Patient A)

Something that buoyed us all up as we all realized this was going to be something that would dramatically improve his quality of life and looked very optimistic even from the start of his trial. (Doctor 2 for Patient C)

However, in some cases, rapid progression of the cancer overshadowed the effectiveness of the intrathecal infusion for managing the pain.

I was hoping that with removal of the pain, which we have achieved, that his quality of life would go up...I am disappointed that that has not happened and it is primarily because of the abdominal muscle spasms and the lymphedema. (Doctor 1 for Patient D)

Some of the expectations were overly optimistic and led to disappointment.

It is challenging because I think patients think it is going to be this wonderful pain free, like I have this little machine and it is all going to be good, but it is not from my limited experience. (Nurse 1 for Patient F)

Comfort level with the device - trust: The concept of trust in building relationships and trust in the device prevailed in the staff's comfort level.

The only way to look after anybody is to assume that the pump is running correctly. (Nurse 2 for Patient C)

We also see a lot more other people involved in their care, not just the nurses and doctors on this unit. (Nurse 2 for Patient D)

I have now come to the point where the intrathecal is just another tool that we use to manage pain. (Nurse 2 for Patient E)

\section{DISCUSSION}

The present study provides some insight into the factors affecting the quality of life of patients requiring intrathecal infusions for intractable pain, and identifies concerns that are useful in planning services. It is the first study to address concerns specific to intrathecal pumps among staff caring for these highly complex patients, particularly with respect to the changing 'culture' on PCUs in the era of the residential hospice option for care. The study took place over a period of recognition of the important role that intrathecal pumps have to play in the care of patients with complex and severe cancer pain. Attitude changes occurred on our unit, which are very likely to be experienced by other PCUs previously unfamiliar with intrathecal pumps. We hope that by sharing 


\section{TABLE 5}

\section{Recommendations}

The palliative care team should have time to establish a relationship with patients and their families before initiating an intrathecal infusion

Patients and their families should have a multidisciplinary psychosocial assessment before the infusion trial, and ongoing psychosocial support afterward

The different disciplines involved in patient care should communicate frequently and clearly, especially in establishing a clear understanding of the rationale for initiating the infusion

Staff should receive education about intrathecal therapy to allay fears about the intervention process, and written care guidelines should be provided Multiprofessional palliative care is required in parallel with specialist pain management

Recognize that an intrathecal infusion requires significant labour resources, both in skills and numbers of staff, and that a program needs to be adequately resourced

Recognize that the initiation of a technical and intensive medical intervention may create stress and conflict among staff in a care setting with a strong noninterventional hospice philosophy

With good planning and cooperation between clinicians and administrators, these obstacles can, and should, be overcome

our experience we can facilitate and ease the transition to more widespread use of intrathecal pumps.

Despite considerable advances in analgesic therapies, such as the use of methadone, lidocaine infusions and ketamine, there will remain a small percentage of unfortunate cancer patients who experience uncontrolled pain and yet are not at the end of life. As palliative chemotherapy treatments improve and the population ages, it is likely that their numbers will increase. The fact that intrathecal infusion may safely and effectively relieve these patients' pain mandates palliative care providers to ensure that their patients have access to intrathecal pumps. Even in the United States, recent reports state that intrathecal infusions are underused despite clear evidence of effectiveness $(24,25)$.

There are many obstacles to cancer patients accessing intrathecal pumps, including the capital costs of the equipment ( $\$ 11,000$ to $\$ 12,000)$, availability of appropriately trained staff, the cost of providing on-call services for small numbers of patients and an inclination among experienced palliative care staff to avoid invasive treatments. Despite the obstacles, the potential benefits are so great that this treatment should be considered an essential part of a comprehensive cancer pain management program. With good planning and cooperation between the care providers and administrators, these obstacles can be overcome.

As a result of the comments made by patients, family members and staff during the present study, we have presented a list of recommendations for programs moving toward making intrathecal infusions available to their patients (Table 5). Communication is a key theme in all the recommendations, both between health care providers and with the patient and family. It should be appreciated that when first referred to a specialist service, patients are usually in a pain crisis, heavily medicated and very stressed. Preinfusion psychosocial assessment may be extremely difficult under these circumstances, and the trial period with a temporary catheter may allow time, not just for optimization of infusion contents and rate, but also for multidisciplinary assessment, education and preparation.
It is very important to accurately convey a clear description of the procedures and after-care requirements involved to the patient and family before the permanent implant, so that expectations are realistic. The high level of distress experienced by nurses caring for these extremely complex patients needs to be recognized. Nurses' educational needs should be met before having to care for a patient with an intrathecal infusion, especially around the time of the initial trial, when the patient requires particularly intense monitoring. When the nurses had cared for the patients before the intrathecal trial and had seen the intensity of their suffering, it was easier for them to understand the rationale for the invasive intervention. Having written instructions is extremely useful, particularly for night shift nurses, who often work with much less support than is available during the day.

Palliative care programs need to work closely with large anesthesia services to be sustainable. A close liaison with local care providers and on-call anesthesia services is vital to reduce patient anxiety about device failure, and dependence on one or two key individuals.

Patients with advanced cancer, especially those who have experienced extreme pain, usually have highly complex ongoing medical and psychosocial needs. Other problems will inevitably arise and may require hospitalization or residential hospice admission. Anesthesia, oncology and palliative care hospital and community teams need to work closely together to fully meet patients' needs as they progress toward the end of life (26).

\section{LIMITATIONS}

The present series was small, including only six patients, but saturation was reached with themes identified in the interviews, suggesting strong similarities between the experiences of all participants. Our unit performs the majority of intrathecal pump insertions in the province, and the study took two years to complete. We implanted 14 pumps and two domes over seven years. A larger study would be unlikely to provide further information, and would be very difficult to complete. A study performed in a larger centre may not provide results applicable to the vast majority of local or regional institutions, either in Canada or in other countries in which use of this technology is rare. Intrathecal infusions in our palliative care programs are offered only for intractable cancer pain - a treatment of last resort. Other centres, particularly those in the United States where intrathecal infusions are more widely used, may have more liberal patient selection criteria, and their experience may differ considerably from ours. We direct this report and these recommendations to palliative care programs without a history of close interaction with anesthesia support.

\section{CONCLUSIONS}

Before having an intrathecal infusion, the pain experience for these patients was debilitating. Intrathecal infusion was seen as a 'last resort' for pain relief and raised hopes of a life without pain. Although the patients' initial expectations were not always met, all participants agreed that their pain had been significantly reduced by the intrathecal infusion and they were pleased to have had access to it, even when complications occurred. Our findings confirmed our impressions that the intrathecal program is a very valuable and much appreciated 
option for the small proportion of highly distressed cancer patients who have exhausted all other analgesic treatments.

Palliative care physicians identified their moral and ethical responsibility to these patients as the catalyst behind the decision to initiate the intrathecal infusion. Accessibility to resources and the patients' ability to make informed decisions were also factored into the decision-making process. Palliative care nurses identified the need for education about intrathecal infusions and good communication among all health care workers as key components to providing care, particularly with respect to the decision to embark on an intrathecal trial. It was clear that, although pain improved with the intrathecal infusion, patients required comprehensive ongoing palliative care afterward. Despite the anxiety induced by the high-acuity care required, it was appropriate that these patients were cared for in a palliative care environment. All staff believed that, whenever possible, it was important for the palliative care team to establish a relationship with the patient and their family

\section{REFERENCES}

1. Hanks GW, Conno F, Cherny N, et al; Expert Working Group of the Research Network of the European Association for Palliative Care. Morphine and alternative opioids in cancer pain: The EAPC recommendations. Br J Cancer 2001;84:587-93.

2. Krames E. Practical issues when using neuraxial infusion. Oncology 1999;13(5 Suppl 2):37-44.

3. Wang JF, Nauss LA, Thomas JE. Pain relief by intrathecally applied morphine in man. Anesthesiology 1979;50:149-51.

4. Nitescu P, Appelgren L, Linder LE, et al. Epidural versus intrathecal morphine-bupivocaine: Assessment of consecutive treatments in advanced cancer pain. J Pain Symptom Manage 1990;5:18-26.

5. Waldman S. Implantable drug delivery systems: Practical considerations. J Pain Symptom Manage 1990;5:169-74.

6. Crul BJP, Delhaas E. Technical complications during long-term subarachnoid or epidural administration of morphine in terminally ill cancer patients: A review of 140 cases. Reg Anesth 1991;16:209-13.

7. Krames E. Intrathecal infusional therapies for intractable pain: Patient management guidelines. J Pain Symptom Manage 1993;8:36-46.

8. Lamer T. Treatment of cancer-related pain: When orally administered medications fail. Mayo Clin Proc 1994;69:473-80.

9. Samuelsson H, Malmberg F, Eriksson M, Hedner T. Outcomes of epidural morphine treatment in cancer pain: Nine years of clinical experience. J Pain Symptom Manage 1995;10:105-12.

10. Aldrete A. Epidural fibrosis after permanent catheter insertion and infusion. J Pain Symptom Manage 1995;10:624-31.

11. Smitt PS, Tsafka A, Teng-van de Zande F, et al. Outcome and complications of epidural analgesia in patients with chronic cancer pain. Cancer 1998;83:2015-22.

12. Naumann C, Erdine S, Koulousakis A, Buyten J-P, Schuchard M. Drug adverse events and system complications of intrathecal opioid delivery for pain: Origins, detection, manifestations, and management. Neuromodulation 1999;2:92-107. before the infusion, as well as to provide ongoing psychosocial support afterwards. There are significant organizational and workload implications resulting from providing intrathecal infusion therapy.

We hope that the present study will help expand access to implantable intrathecal infusion pumps for people with intractable cancer pain, and that the specific recommendations (Table 5) are useful in achieving this goal.

ACKNOWLEDGEMENTS: Dr Hawley dedicates this article to Helen, in memory of Amy.

This work was presented at the Canadian Society of Hospice Palliative Care Association annual meeting in Toronto, Ontario, November 2007. The study was supported financially by private donations to the Vancouver Cancer Centre Palliative Care Fund of the BC Cancer Foundation. None of the authors have received any financial or material benefits from Medtronic Inc.

13. Mercadante S. Problems of long-term spinal opioid treatment in advanced cancer patients. Pain 1999;79:1-13.

14. Anderson V, Burchiel K, Cooke B. A prospective, randomized trial of intrathecal injection vs epidural infusion in the selection of patients for continuous intrathecal opioid therapy. Neuromodulation 2003;6:142-52.

15. Rauck R, Cherry D, Boyer M, et al. Long-term intrathecal opioid therapy with a patient-activated implanted delivery system for the treatment of refractory cancer pain. J Pain 2003;4:441-7.

16. Mercadante S, Intravaia G, Villari P, et al. Intrathecal treatment in cancer patients unresponsive to multiple trials of systemic opioids. Clin J Pain 2007;23:793-8.

17. Smith TJ, Staats PS, Deer T, et al. Randomized clinical trial of an implantable drug delivery system compared with comprehensive medical management for refractory cancer pain: Impact on pain, drug-related toxicity, and survival. J Clin Oncol 2002;20:4040-9.

18. Portenoy R. Managing pain in patients with advanced cancer: The role of neuraxial infusion. Oncology 1999;13(5 Suppl 2):7-8.

19. Christo P, Mazloomdost D. Interventional pain treatments for cancer pain. Ann NY Acad Sci 2008;1138:299-328.

20. Du Pen S. Complications of neuraxial infusion in cancer patients. Oncology 1999;13(5 Suppl 2):45-51.

21. Stearns L, Boortz-Marx R, Du Pen S, et al. Intrathecal drug delivery for the management of cancer pain. J Supp Oncol 2005;1:399-408.

22. Hearn J, Higginson IJ. Development and validation of a core outcome measure for palliative care: The Palliative Outcome Scale. Qual Health Care 1999;8:219-27.

23. Palliative outcome scale (POS). <http://www.kcl.ac.uk/schools/ medicine/depts/palliative/qat/pos.html $>$ (Version current at October 5, 2009)

24. Brogan S. Intrathecal therapy for the management of cancer pain. Curr Pain Headache Rep 2006;10:254-9.

25. Smith T, Swainey C, Coyne P. Pain management, including intrathecal pumps. Curr Pain Headache Rep 2004;6:291-6.

26. Staats P. Neuraxial infusion for pain control: When, why and what to do after the implant. Oncology 1999;13(5 Suppl 2):58-62. 


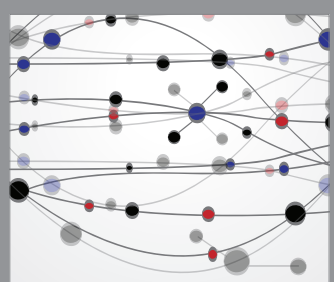

The Scientific World Journal
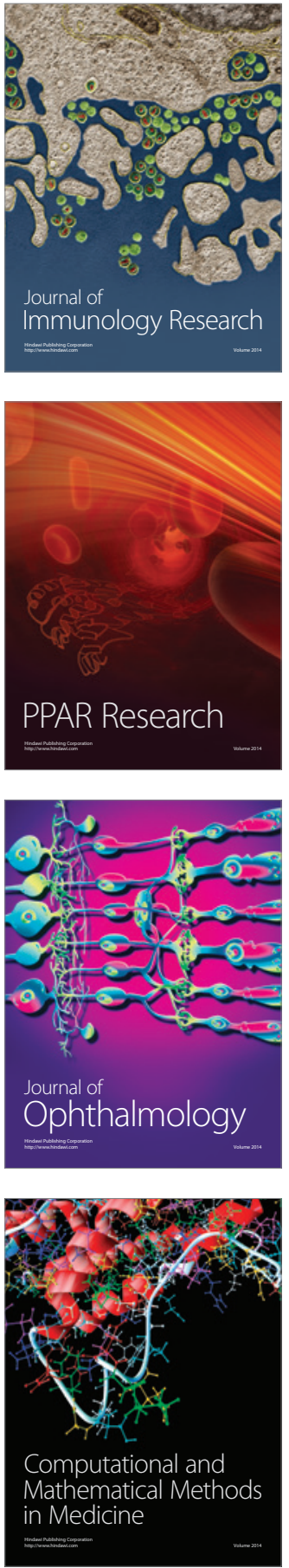

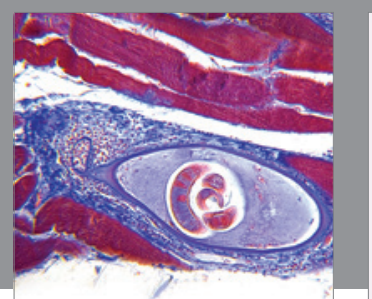

Gastroenterology Research and Practice

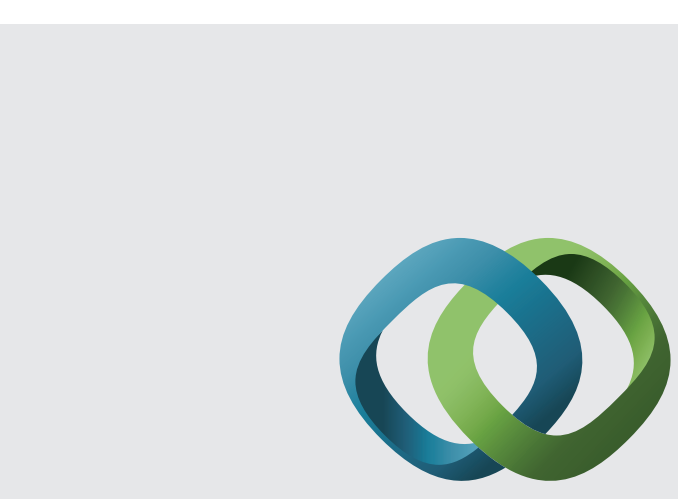

\section{Hindawi}

Submit your manuscripts at

http://www.hindawi.com
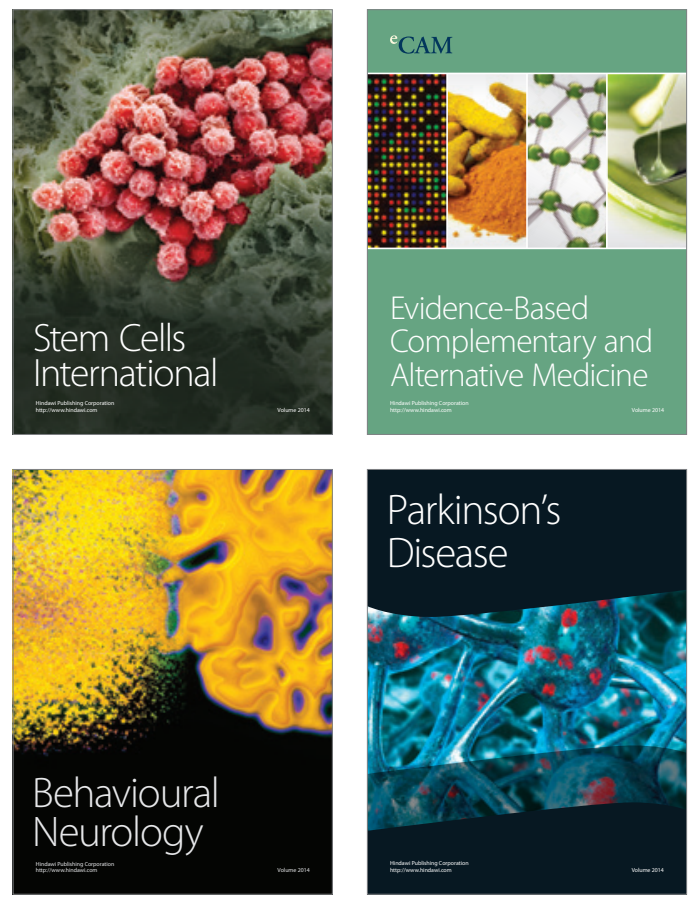
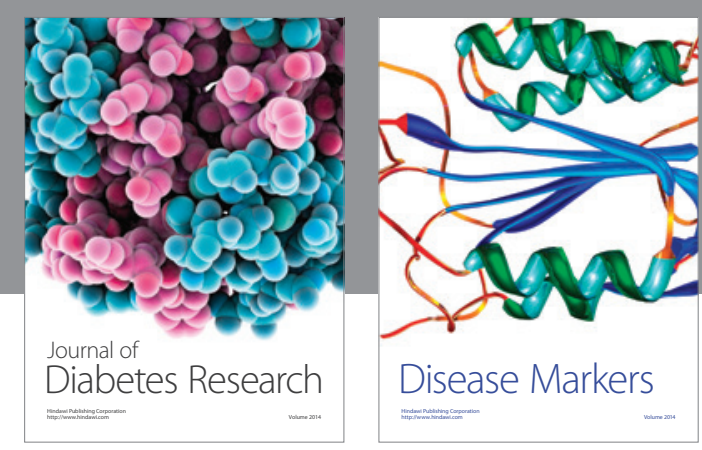

Disease Markers
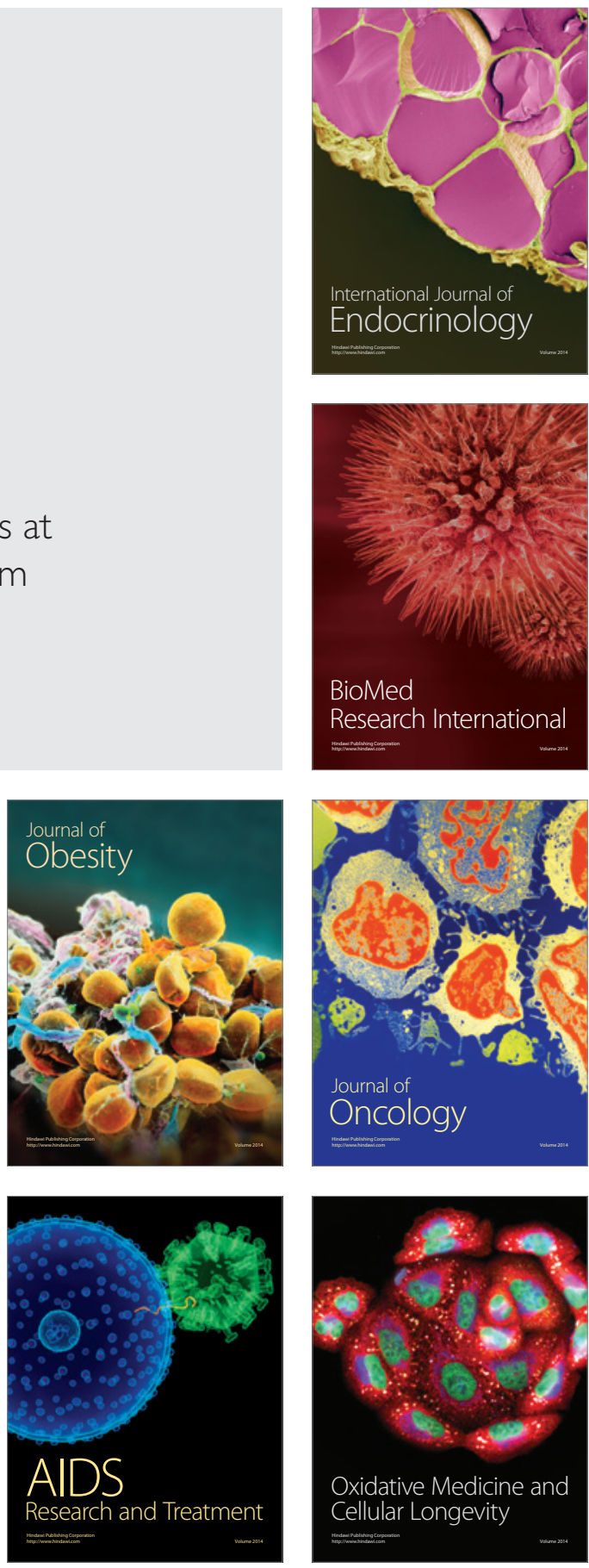